\title{
Unusual Postoperative Pouch Related Complication in Two Ulcerative Colitis Cases
}

\author{
Yuki Hiramoto ${ }^{1}$, Hidejiro Kawahara ${ }^{1}$, Mitsumasa Takeda ${ }^{1}$, Tomo Matsumoto ${ }^{1}$, Takeyuki Misawa ${ }^{1}$, \\ Katsuhiko Yanaga ${ }^{2}$
}

\author{
Corresponding author: \\ Hidejiro Kawahara, M.D. \\ Department of Surgery \\ Kashiwa Hospital, Jikei University \\ School of Medicine, \\ 163-1 Kashiwashita, Kashiwashi, \\ Chiba277-8567, Japan \\ Tel: +81-4-7164-1111 Ext. 3421, \\ Fax: +81-4-7163-3488 \\ E-mail: kawahide@jikei.ac.jp
}

Received: 18.02 .2019

Accepted: 20.03.2019

Copyright () Celsius Publishing House www.sgo-iasgo.com
'Department of Surgery, Kashiwa Hospital, Jikei University School of Medicine, Chiba, Japan 2Department of Surgery, Jikei University School of Medicine, Tokyo, Japan

\section{ABSTRACT}

A restorative proctocolectomy with ileal pouch-anal anastomosis (IPAA) has been considered a gold standard surgical procedure for patients with ulcerative colitis (UC). Even if patients with UC underwent high quality IPAA after proctocolectomy, pouch related complications occurred in over $50 \%$ of them more than five years after surgery. The most common pouch related complication is pouchitis including frequent bowel movements, urgency, cramping and bloody stools. We experienced unusual postoperative pouch related complication, pouch inertia, in two female patients over ten years after IPAA. Pouch inertia is the state that pouch is expanded due to losing shrinkage ability. It is considered one of pouch failure however it remains unknown. The patient who underwent pouch resection has been fine after the surgery, but the other patient who did not undergo pouch resection by her decision-making died. Because a pouch inertia is a frequent cause of life-threatening disease, pouch resection should be performed for patients with pouch inertia as soon as possible. We report unusual postoperative pouch related complication in two ulcerative colitis cases.

Key words: ulcerative colitis, pouchitis, pouch inertia

\section{INTRODUCTION}

Ulcerative colitis (UC) is a chronic disease featuring recurrent inflammation of the colonic mucosa. About $20 \%-25 \%$ of patients with UC require a colectomy at some point in their lives, and in most cases the operative surgical procedure chosen is a restorative proctocolectomy with ileal pouch-anal anastomosis (IPAA) $(1,2)$. Although diseased colonic mucosa is removed during the procedure, and transanal fecal continence is maintained by creating an ileal pouch, patients may develop acute or chronic inflammation of the pouch, so called pouchitis. Pouchitis is the most common complication of IPAA after proctocolectomy for UC, occurring in over $50 \%$ of patients experiencing an acute episode within 5 years and about $5 \%$ of those going on to develop chronic inflammation (1-5), even if high quality IPAA after proctocolectomy was performed. We experienced unusual postoperative pouch related complication, pouch inertia, in two female 
patients over ten years after proctocolectomy. Pouch inertia is the state that pouch is expanded due to losing shrinkage ability. It is considered one of pouch failure however it remains unknown (6). The term has not been clearly defined yet, and there are few reports about it.

\section{CASE PRESENTATION 1}

35-year-old female underwent proctocolectomy with ileal pouch-anal anastomosis in our hospital because of severe ulcerative colitis in 2002. She had received medication regularly as outpatient since then. She had been provided conservative treatment due to severe pouchitis since 2007 (five years after surgery). She had repeated hospitalization in our hospital due to dehydration caused by severe pouchitis since 2010 (eight years after surgery). Computed tomography demonstrated her pouch developed like mega-cyst with a large quantity of bowel contents occupied her pelvic cavity (fig. 1). She could not discharge enough bowel contents through the anus. We strongly recommended her to receive surgical treatment, pouch resection, because of decreasing oral intake and body weight loss, however she did not decide to receive pouch resection because pouch resection means receiving eternal ileostomy. She disgusted with receiving eternal ileostomy strongly. She was infected with cytomegalovirus subsequently. The cytomegalovirus infection was cured by adequate conservative treatments. In February 2015, she was suddenly carried into the emergency room in our hospital because of cardiac arrest caused by hyperkalemia. We could not rescue her even though she was provided any appropriate treatments.

\section{CASE PRESENTATION 2}

31-year-old female underwent proctocolectomy with ileal pouch-anal anastomosis in our hospital due to severe ulcerative colitis in 2002. She had received medication regularly as outpatient since then. She had been provided conservative treatment due to severe pouchitis since 2007 (five years after surgery). She had repeated hospitalization in our hospital due to dehydration caused by severe pouchitis since 2012 (ten years after surgery). Computed tomography demonstrated her pouch developed like mega-cyst with a large quantity of bowel contents occupied her

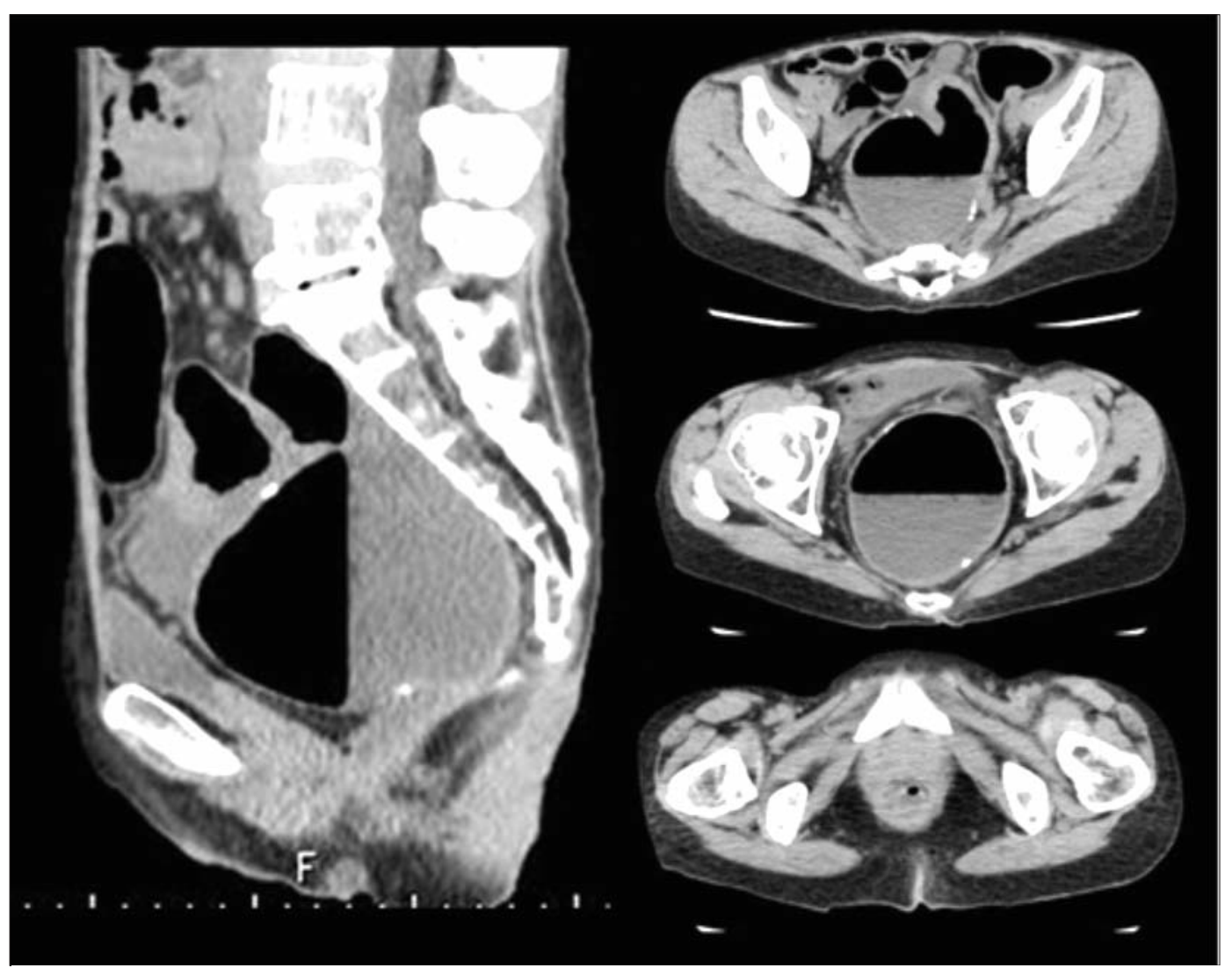

Figure 1 - Computed tomography demonstrated her pouch developed like mega-cyst with a large quantity of bowel contents occupied her pelvic cavity in case 1 


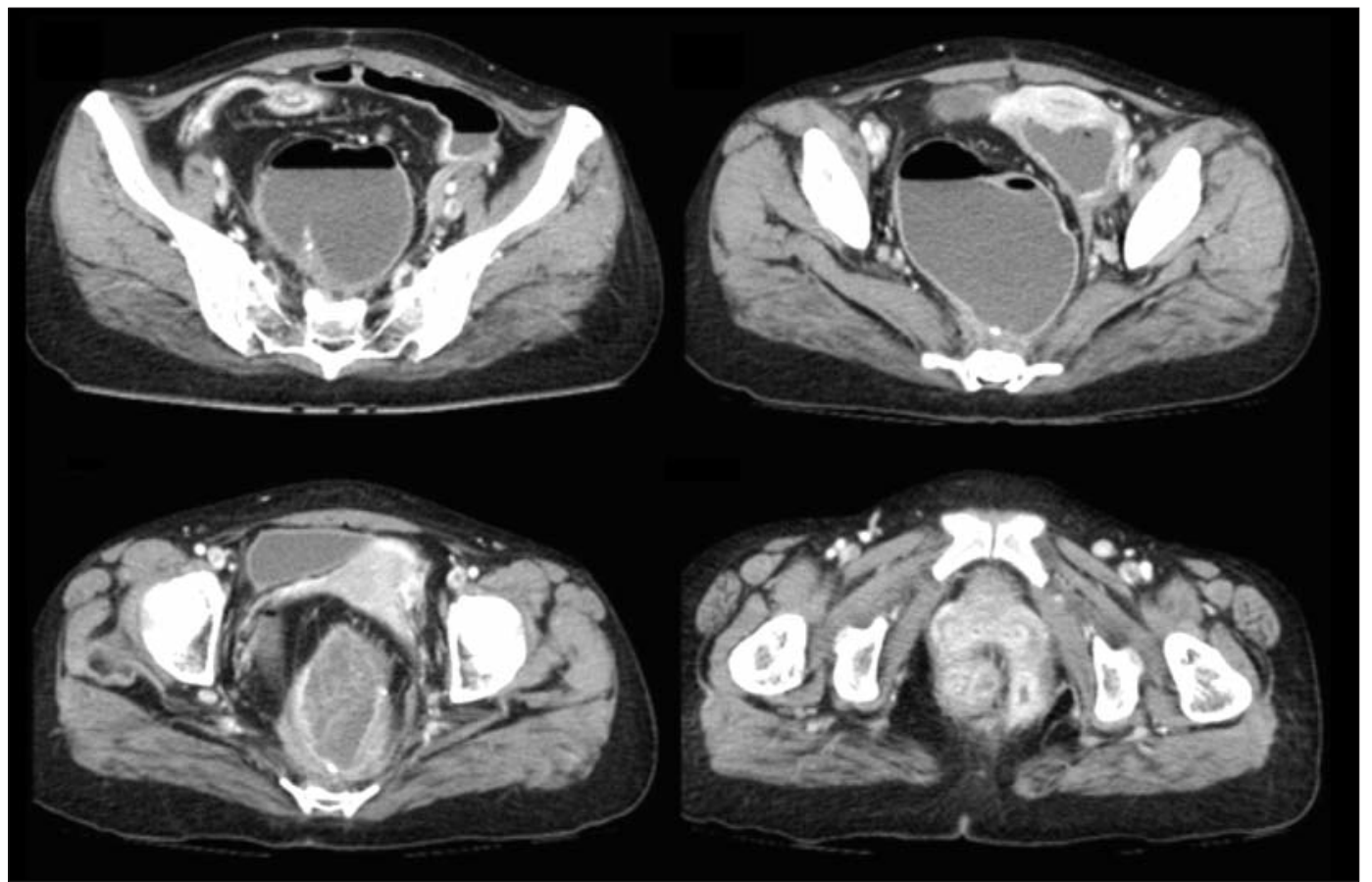

Figure 2 - Computed tomography demonstrated her pouch developed like mega-cyst with a large quantity of bowel contents occupied her pelvic cavity in case 2

pelvic cavity (fig. 2). She could not discharge enough bowel contents through the anus. We recommended her to receive surgical treatment, pouch resection, because of decreasing oral intake and severe emaciation. In September 2014, she underwent pouch resection with eternal ileostomy. She has been fine since then with no problem.

\section{DISCUSSION}

A restorative proctocolectomy with IPAA has been considered a gold standard surgical procedure for patients with UC. Laparoscopic proctocolectomy with IPAA has been performed for UC since 1992 reported by Peters (7), while it has been introduced since 2001 at our institution by an author of this report (H.K)(8). And we reported short-term outcome of patients who underwent proctocolectomy with IPAA during 13 years between 2000 and 2012. It concluded that proctocolectomy with IPAA by both open and laparoscopic approaches were feasible and acceptable (8). In our institution, approximately eight patients with UC have undergone this procedure every each year since 2000 until now. Pouchitis was encountered in some of those patients after surgery. Pouchitis is the most common complication of IPAA after total proctocolectomy for UC, occurring in over $50 \%$ of patients at some time after IPAA (1-5). The most common symptoms include frequent bowel movements, urgency, cramping and bloody stools (9). Pouchitis is a chronic inflammatory condition limited to ileal pouch. Pouch with severe inflammation was shrinkage or stenotic shaped intestine demonstrated by computed tomography or colonoscopy. It is extremely rare that pouch developed like mega-cyst due to inflammation.

In this report, the two females could not discharge enough bowel contents through the anus due to pouch inertia. Pouch inertia caused not only decreasing oral intake and body weight loss but also to be compromised host, life-threatening disease. Antibiotic therapy is a mainstay of treatment for pouchitis. In cases of antibiotic resistance, therapeutic interventions may escalate to include biologics. Failed medical therapy may require pouch resection with ileostomy (10).

The patient who underwent pouch resection has been fine after the surgery, but the other patient who did not undergo pouch resection by her decisionmaking died. Health-related quality of life in patients operated for rectal cancer with permanent stoma was inferior compared to patients without stoma. Almost all patients disgusted with receiving eternal stoma before surgery (11). She also strongly disgusted with receiving eternal ileostomy, however we regretted that she could not be persuaded to receive pouch resection.

Because a pouch inertia is a frequent cause of life- 
threatening disease, pouch resection should be performed for patients with pouch inertia as soon as possible.

\section{Conflict of Interest Statement}

The Authors declare that there are no conflicts of interest regarding this study.

\section{REFERENCES}

1. Onaitis MW, Mantyh C. Ileal pouch-anal anastomosis for ulcerative colitis and familial adenomatous polyposis: historical development and current status. Ann Surg. 2003;238:S42-8.

2. Fazio VW, Ziv Y, Church JM, Oakley JR, Lavery IC, Milsom JW, Schroeder TK. Ileal pouch-anal anastomoses complicationsand function in 1005 patients. Ann Surg. 1995;222:120-7.

3. Sandborn WJ, Pardi DS. Clinical management of pouchitis. Gastroenterology. 2004;127:1809-14

4. Hurst RD, Molinari M, Chung TP, Rubin M, MichelassiF. Prospective study of the incidence, timing and treatmentof pouchitis in 104 consecutive patients after restorativeproctocolectomy. Arch Surg
1996;131:497-500; discussion 501-2.

5. Svaninger G, Nordgren S, Oresland T, Hultén L. Incidenceand characteristics of pouchitis in the Kock continent ileostomyand the pelvic pouch. Scand J Gastroenterol. 1993;28:695-700.

6. Ikeuchi H, Uchino M, Sugita A, Futami K, Fukushima K, Hata K, et al. Long-term outcomes following restorative proctocolectomy ileal pouch-anal anastomosis in pediatric ulcerative colitis patients: Multicenter national study in Japan. Ann Gastroenterol Surg. 2018; 2:428-433.

7. Peters WR. Laparoscopic total proctocolectomy with creation of ileostomy for ulcerative colitis: report of two cases. J Laparoendosc Surg. 1992;2:175-8.

8. Watanabe K, Kawahara H, Tomoda M, Hojo S, Akiba T, Yanaga K. Surgical challenge for ulcerative colitis at Kashiwa Hospital, the Jikei university school of medicine. Jikeikai Med J. 2015; 64:89-93.

9. Sandborn WJ Pouchitis following ileal pouch-anal anastomosis: definition, pathogenesis, and treatment. Gastroenterology. 1994;107: $1856-60$.

10. Angriman I, Scarpa M, Castagliuolo I. Relationship between pouch microbiota and pouchitis following restorative proctocolectomy for ulcerative colitis.World J Gastroenterol. 2014;20:9665-74.

11. Näsvall P, Dahlstrand U, Löwenmark T, Rutegard J, Gunnarsson U, Strigard K.Quality of life in patients with a permanent stoma after rectal cancer surgery. Qual Life Res. 2017;26:55-64. 